\title{
A Quantum-Chemical Approach to Develop Tetrahydroquinoxaline as Potent Ferroptosis Inhibitors
}

\author{
Hong-Xu Lei ${ }^{1}$, KaiLi Zhang ${ }^{2}$, Yu-Xi Qin ${ }^{1}$, rongjian dong ${ }^{3}$, Dezhan Chen ${ }^{1}$, HaiFeng Zhou ${ }^{2}$, \\ and xiehuang sheng ${ }^{1}$ \\ ${ }^{1}$ Shandong Normal University \\ ${ }^{2}$ China Three Gorges University \\ ${ }^{3}$ Pingyang center for food and drug control, Wenzhou
}

July 7, 2020

\begin{abstract}
Ferroptosis is a recently characterized form of regulated necrosis with the iron-dependent accumulation of (phospho)lipid hydroperoxides $(\mathrm{LOOH})$. It has attracted considerable attention for its putative involvement in diverse pathophysiological processes, such as cardiovascular disease and neurodegeneration. Here we describe the discovery of tetrahydroquinoxaline, a novel scaffold of ferroptosis inhibitors based on quantum chemistry methods. Tetrahydroquinoxaline deviates showed very good inhibition of ferroptosis, while being non cytotoxic for human cancer cells. And, the advantage of them is their small molecular weight $(\mathrm{MW} .=148 \mathrm{Da})$ that can be coupled with other drugs to form multi-target drugs to better meet the treatment of complicated diseases.
\end{abstract}

\section{Hosted file}

main article.docx available at https://authorea.com/users/339561/articles/467705-a-quantumchemical-approach-to-develop-tetrahydroquinoxaline-as-potent-ferroptosis-inhibitors 